\title{
On the performance of the moment approximation for the numerical computation of fiber stress in turbulent channel flow
}

\author{
J. J. J. Gillissen and B. J. Boersma \\ Laboratory for Aero and Hydrodynamics, J.M. Burgers Center, Leeghwaterstraat 21, \\ 2628 CA Delft, The Netherlands \\ P. H. Mortensen and H. I. Andersson \\ Department of Energy and Process Engineering, Norwegian University of Science and Technology, \\ 7491 Trondheim, Norway
}

(Received 3 July 2006; accepted 9 January 2007; published online 8 March 2007)

\begin{abstract}
Fiber-induced drag reduction can be studied in great detail by means of direct numerical simulation [J. S. Paschkewitz et al., J. Fluid Mech. 518, 281 (2004)]. To account for the effect of the fibers, the Navier-Stokes equations are supplemented by the fiber stress tensor, which depends on the distribution function of fiber orientation angles. We have computed this function in turbulent channel flow, by solving the Fokker-Planck equation numerically. The results are used to validate an approximate method for calculating fiber stress, in which the second moment of the orientation distribution is solved. Since the moment evolution equations contain higher-order moments, a closure relation is required to obtain as many equations as unknowns. We investigate the performance of the eigenvalue-based optimal fitted closure scheme [J. S. Cintra and C. L. Tucker, J. Rheol. 39, 1095 (1995)]. The closure-predicted stress and flow statistics in two-way coupled simulations are within 10\% of the "exact" Fokker-Planck solution. (C) 2007 American Institute of Physics. [DOI: 10.1063/1.2437824]
\end{abstract}

\section{INTRODUCTION}

It is well known that the addition of a small amount of long-chained flexible polymers to a turbulent pipe flow can induce changes in the turbulent structures, leading to a reduction of the drag. ${ }^{1}$ Similar but less profound effects have also been observed using stiff polymers ${ }^{2}$ and macroscopic slender particles. ${ }^{3}$ An extensive overview with respect to drag reduction can be found in Ref. 4.

It is a generally accepted assumption that the key properties of a polymeric liquid are elasticity and viscous anisotropy. Elasticity originates from the ability of polymers to stretch and recoil, introducing a mechanism of energy transfer between the polymers and the fluid. Viscous anisotropy, or a directional-dependent viscosity, is the result of the anisotropic distribution of polymer orientation angles.

The physical mechanisms underlying polymer-induced drag reduction are not well understood and numerical tools have widely been used to gain deeper insight. Most numerical work on polymer-induced drag reduction is based on computing the additional stress generated by the polymers, which is added to the Navier-Stokes equations. Commonly, the constitutive models for the stress tensor are expressions in moments of the statistical distribution of polymer configuration. Due to the nonlinear nature of polymer motion, moment evolution equations suffer from a closure problem. In this paper, we study the closure problem involved in the computation of the stress in a suspension of rigid rod-like polymers, referred to as fibers.

In contrast to flexible polymers, numerical simulations of turbulent fiber suspensions are rare. To the authors' knowledge only the Stanford group has published on so-called two- way coupled turbulent flow simulations, in which the fiber dynamics are solved as well as the interaction between the fibers and the fluid (Paschkewitz et al. ${ }^{5}$ ). Their method is based on a moment equation derived using a closure. They find that Brownian motion has an adverse effect on drag reduction. Contributions of the different stress components to the momentum and energy equations are studied in detail. Drag reduction is found to originate from the damping of the near wall vortices by cross-stream fiber alignment in the biextensional intervortex regions.

Instead of solving fiber moments, a more direct approach is to solve the complete distribution function, which is governed by the Fokker-Planck equation. This method is "exact," since it does not require a closure relation. On the other hand, it requires much more computational resources as compared to the moment approximation. Numerical solutions of the distribution function can be obtained by computing the trajectories and orientations of individual particles. Manhart adopted such a method, and studied the influence of particle aspect ratio and Brownian motion on fiber stress. ${ }^{6}$ He finds that the rheological properties deviate from Newtonian with increasing aspect ratio $r$, and for very large aspect ratio $r>100$ the properties remain unchanged. Furthermore, with increasing Brownian diffusion, the mean of the stress increases and the standard deviation of the stress decreases.

In this study the spatial and orientational operators in the Fokker-Planck equation are discretized to obtain the fiber distribution function in turbulent channel flow. These solutions are used to investigate the performance of the eigenvalue-based optimal fitted (EBOF) closure $^{7}$ used in the evolution equation for the second moment of this function. 
To fully study the effect of the closure on the fluid mechanics, two-way coupled simulations are performed. A brief comparison in Newtonian turbulent channel flow has been reported earlier in Ref. 8. The closure-predicted mean stress was found to differ up to $30 \%$ from the Fokker-Planck solutions taken from Ref. 6.

The paper is organized as follows. In Sec. II the theory for the stress in a fiber suspension is explained. Section III deals with the numerical procedure for calculating the fiber distribution function. Then, in Sec. IV the moment approximation and the involved closure problem are discussed. In Sec. V the numerical details for solving the turbulent fiber suspension channel flow are given. Results from the moment approximation and the Fokker-Planck equation are compared in Sec. VI. Conclusions are given in Sec. VII. In the Appendix the EBOF closure is explained.

\section{CONSTITUTIVE EQUATIONS}

An extensive treatment of the theory given in this section can by found in Ref. 9. We consider Brownian rigid rods, which are homogeneously suspended in a Newtonian solvent. The fiber volume fraction is $c$ and the solvent viscosity is $\mu$. The fibers are monodispersed, inertia-free, neutrally buoyant, noninteracting and much smaller than the length scale over which the fluid velocity gradient changes. The flow around an individual fiber is governed by Stokes' equations. Under these assumptions the fiber center of mass $\boldsymbol{x}$ and the fiber orientation unit vector $\boldsymbol{p}$ evolve according to

$$
\dot{\boldsymbol{x}}=\boldsymbol{u}-d_{s} \boldsymbol{\nabla} \ln f, \quad \dot{\boldsymbol{p}}=\boldsymbol{\kappa} \cdot \boldsymbol{p}-\boldsymbol{\kappa}: \boldsymbol{p p p}-d_{r} \boldsymbol{\nabla}_{\boldsymbol{p}} \ln f,
$$

where the effect of finite $r$ has been neglected. We only consider the behavior in the limit of infinite $r$. Here $\boldsymbol{u}$ is the local fluid velocity, $\boldsymbol{\kappa}=(\boldsymbol{\nabla} \boldsymbol{u})^{T}$ is the transpose of the local fluid velocity gradient, $\boldsymbol{\nabla}$ is the gradient operator in physical space, $\nabla_{p}$ is the gradient operator on the unit sphere, $d_{s}$ is the spatial diffusion rate, and $d_{r}$ is the rotational diffusion rate.

The distribution function $f(\boldsymbol{p}, \boldsymbol{x}, t)$ gives the probability of finding a fiber with orientation $\boldsymbol{p}$ at position $\boldsymbol{x}$ and time $t$. Since the fibers are homogeneously suspended, the sum of the orientation probabilities is independent of position and time and set to one,

$$
\int d \boldsymbol{p} f=1 \text {. }
$$

The evolution of $f$ is described by the Fokker-Planck equation,

$$
\frac{\partial f}{\partial t}+\nabla \cdot(\dot{\boldsymbol{x}} f)+\nabla_{\boldsymbol{p}} \cdot(\dot{\boldsymbol{p}} f)=0 .
$$

Together with the equations of fiber motion (1) the FokkerPlanck equation reads

$$
\begin{aligned}
\frac{\partial f}{\partial t} & +\boldsymbol{\nabla} \cdot(\boldsymbol{u} f)+\nabla_{\boldsymbol{p}} \cdot([\boldsymbol{\kappa} \cdot \boldsymbol{p}-\boldsymbol{\kappa}: \boldsymbol{p p p}] f)-d_{s} \nabla^{2} f-d_{r} \nabla_{\boldsymbol{p}}^{2} f \\
& =0 .
\end{aligned}
$$

Function $f$ determines the additional stress $\tau$ in the suspension generated by the fibers. In the limit of $r \rightarrow \infty$,

$$
\boldsymbol{\tau}=\alpha \mu\left[2 \boldsymbol{\kappa}:\langle\boldsymbol{p p p p}\rangle+6 d_{r}\left(\langle\boldsymbol{p p}\rangle-\frac{1}{3} \boldsymbol{\delta}\right)\right],
$$

where $\langle\cdots\rangle$ signifies the orientational average over $f$,

$$
\langle\cdots\rangle=\int d p f \cdots,
$$

$\alpha$ is the concentration parameter,

$$
\alpha \sim \frac{c r^{2}}{\ln (r)},
$$

and $\boldsymbol{\delta}$ is the unit tensor. Two stress contributions can be distinguished in Eq. (5). The viscous stress $\alpha \mu 2 \kappa:\langle p p p p\rangle$ equals the projection of the Newtonian viscous stress on to the fiber directional vectors. The elastic stress $6 \alpha \mu d_{r}(\langle p \boldsymbol{p}\rangle$ $\left.-\frac{1}{3} \boldsymbol{\delta}\right)$ originates from the Brownian tendencies to move the distribution towards isotropy.

\section{NUMERICAL METHOD FOR THE FOKKER-PLANCK EQUATION}

\section{A. Galerkin's method}

Our numerical solution procedure for the orientation part of the Fokker-Planck equation (4) utilizes Galerkin's principle with the spherical harmonics as the trial functions. The technique has been used for the case of simple shear in Ref. 10. For simplicity we use a short notation for the rotational advection operator in Eq. (4): $\boldsymbol{\nabla}_{\boldsymbol{p}} \cdot\left(\left[\boldsymbol{e}_{i} \boldsymbol{e}_{j} \cdot \boldsymbol{p}-\boldsymbol{e}_{i} \boldsymbol{e}_{j}: \boldsymbol{p p p}\right] f\right)$ $=M_{i j} f$, where the $\boldsymbol{e}_{i}$ 's denote the unit vectors of the velocity gradient tensor. Without the spatial derivative terms, Eq. (4) then reads

$$
\frac{\partial f}{\partial t}+\sum_{i, j} \kappa_{i j} M_{i j} f-d_{r} \nabla_{p}^{2} f=0 .
$$

Function $f$ is expanded in spherical harmonics up to degree $N$,

$$
f^{N}=\sum_{l=0}^{N} \sum_{m=-l}^{l} A_{l}^{m} f_{l}^{m},
$$

with $A_{l}^{m}$ the expansion coefficients and $f_{l}^{m}$ the spherical harmonics of degree $l$ and order $m$ (Ref. 11). The series expansion is inserted into Eq. (8), giving

$$
\begin{aligned}
& \frac{\partial}{\partial t} \sum_{l, m} A_{l}^{m} f_{l}^{m}+\sum_{i, j} \kappa_{i j} M_{i j} \sum_{l, m} A_{l}^{m} f_{l}^{m}+d_{r} \sum_{l, m} l(l+1) A_{l}^{m} f_{l}^{m} \\
& \quad=\mathfrak{R}^{N} .
\end{aligned}
$$

Here we have used that $\nabla_{p}^{2} f_{l}^{m}=-l(l+1) f_{l}^{m}$. Due to the finite truncation the right-hand side (rhs) of Eq. (10) is not zero but equal to the residual function $\mathfrak{R}^{N}$. The $A_{l}^{m}$ are obtained using Galerkin's method by setting the residual function orthogonal to all basis functions $f_{p}^{q}$ up to degree $N$. Using the orthogonality property of the spherical harmonics, this yields

$$
\frac{\partial A_{p}^{q}}{\partial t}+\sum_{i, j} \kappa_{i j} \sum_{l, m}\left\langle f_{p}^{q} \mid M_{i j} f_{l}^{m}\right\rangle A_{l}^{m}+d_{r} p(p+1) A_{p}^{q}=0,
$$

where the inner product is defined as $\langle a \mid b\rangle=\int d p a b$. The coefficients $\left\langle f_{p}^{q} \mid M_{i j} f_{l}^{m}\right\rangle$ are computed numerically using Gauss- 

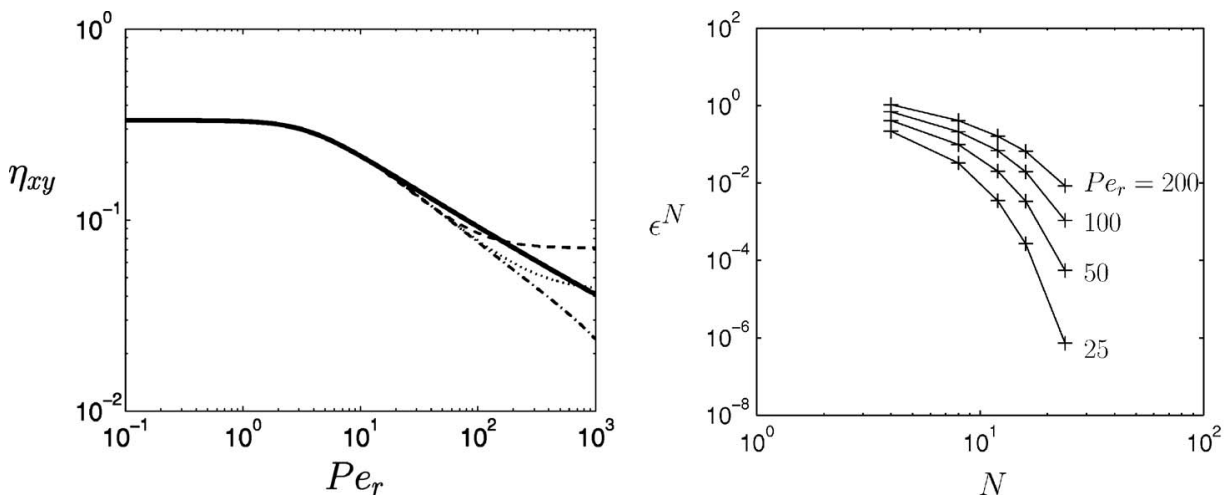

FIG. 1. Left: Shear viscosity [Eq. (14)] vs rotary Peclet number computed with the Fokker-Planck equation (-) and the moment equation, derived with EBOF50 (--), EBOF200 ( $\cdots)$, and EBOF800 (-.). Right: Truncation error [Eq. (15)] vs order of approximation and rotary Peclet number for simple shear.
Legendre integration. ${ }^{12}$ The normalization condition [Eq. (2)] requires $A_{0}^{0}=1$, and point symmetry $f(-\boldsymbol{p})=f(\boldsymbol{p})$ sets all coefficients $A_{l}^{m}$ for odd $l$ to zero.

\section{B. Accuracy}

Here we study the accuracy of the method outlined above for the case of simple shear,

$$
\boldsymbol{\kappa}=\left(\begin{array}{lll}
0 & \gamma & 0 \\
0 & 0 & 0 \\
0 & 0 & 0
\end{array}\right),
$$

with $\gamma$ the shear rate. The stationary solution to Eqs. (11) and (12), obtained using a lower upper decomposition, depends on two parameters: the order of truncation $N$ and the rotary Peclet number $\mathrm{Pe}_{r}$. This number is defined as the ratio of the characteristic shear and the rotary diffusion coefficient,

$$
\mathrm{Pe}_{r}=\frac{\sqrt{\frac{1}{2}\left(\boldsymbol{\kappa}+\boldsymbol{\kappa}^{T}\right)^{2}}}{d_{r}} .
$$

When suspended in shear flow, fibers tend to align in the streamwise direction. The level of fiber alignment is controlled by Brownian motion, which smears out the distribution function. With increasing $\mathrm{Pe}_{r}$ (diminishing rotary diffusion), $f$ evolves from isotropic to localized in the streamwise direction. Since in that direction there is no strain, an increase in alignment results in a decrease in shear stress. This so-called shear thinning phenomenon is illustrated in Fig. 1, showing the shear viscosity $\eta_{x y}$ as a function of $\mathrm{Pe}_{r}$,

$$
\eta_{x y}=\frac{\tau_{x y}}{\alpha \mu \gamma} .
$$

In Fig. 1 we also show the truncation error $\epsilon^{N}$, which is defined as

$$
\epsilon^{N}=\sqrt{\frac{\int d \boldsymbol{p}\left(f^{N}-f\right)^{2}}{\int d \boldsymbol{p} f^{2}}} .
$$

Here we approximated the exact solution $f$ with $f^{48}$, which for this case gave almost identical results as with $f^{64}$. With increasing $\mathrm{Pe}_{r}$, the peak width of $f$ decreases and more modes are needed to accurately approximate this shape. Accordingly, the value of $N$ required for an accurate solution increases with $\mathrm{Pe}_{r}$. This dependence is used in choosing the values for $\mathrm{Pe}_{r}$ and $N$ in the turbulent channel flow simulations described in Sec. V.

\section{MOMENT APPROXIMATION}

\section{A. Moment evolution equations}

The distribution of fiber orientation depends both on spatial and orientation coordinates [Eq. (4)]. As a consequence numerical computations are very expensive. However, the fiber stress depends solely on its second- and fourth-order moments $\langle\boldsymbol{p} \boldsymbol{p}\rangle$ and $\langle\boldsymbol{p} \boldsymbol{p} \boldsymbol{p} \boldsymbol{p}\rangle$. A direct computation of these moments by solving the corresponding transport equations reduces computational costs considerably. The moment transport equations are derived by multiplying the FokkerPlanck equation (4) by $\boldsymbol{p p}$ (or $\boldsymbol{p p p p}$, etc.) and subsequently integrating over the orientation angles. By this procedure the evolution equation for the second moment is found to be ${ }^{9}$

$$
\begin{gathered}
\frac{\partial\langle\boldsymbol{p} \boldsymbol{p}\rangle}{\partial t}+\boldsymbol{u} \cdot \boldsymbol{\nabla}\langle\boldsymbol{p} \boldsymbol{p}\rangle-\boldsymbol{\kappa} \cdot\langle\boldsymbol{p} \boldsymbol{p}\rangle-\langle\boldsymbol{p} \boldsymbol{p}\rangle \cdot \boldsymbol{\kappa}^{T}+2 \boldsymbol{\kappa}:\langle\boldsymbol{p p p p}\rangle \\
-d_{s} \nabla^{2}\langle\boldsymbol{p} \boldsymbol{p}\rangle-6 d_{r}\left(\frac{1}{3} \boldsymbol{\delta}-\langle\boldsymbol{p} \boldsymbol{p}\rangle\right)=0 .
\end{gathered}
$$

The unknown fourth moment $\langle p p p p\rangle$ appears in the equation of change for the second moment. In fact, all moment evolution equations contain higher-order moments and the system of moment evolution equations can never be closed. This is a consequence of the nonlinear dependence of fiber rotation on fiber orientation [Eq. (1)]. To obtain a closed set of moment equations an ad hoc relation must be adopted to express a higher-order moment in terms of a lower moment. The error introduced by this so-called closure relation is the subject of the present study.

\section{B. Closure}

In terms of the expansion [Eq. (9)] the closure relates $A_{4}^{m}$ to $A_{2}^{m}$. Since in the $\langle\boldsymbol{p} \boldsymbol{p}\rangle$-principal frame there are three nonzero $A_{4}^{m}$ and two nonzero $A_{2}^{m},\langle\boldsymbol{p} \boldsymbol{p} \boldsymbol{p} \boldsymbol{p}\rangle$ is related to $\langle\boldsymbol{p} \boldsymbol{p}\rangle$ through three scalar functions depending on two scalar arguments. $^{7}$ These relations can be constructed by parametrizing the distribution function ${ }^{13}$ or by fitting exact solutions of the Fokker-Planck equation. ${ }^{7,14}$ In this work, we study the EBOF closure proposed by Cintra and Tucker. ${ }^{7}$ It is an expression in eigenvalues of $\langle\boldsymbol{p p p p}\rangle$ and $\langle\boldsymbol{p} \boldsymbol{p}\rangle$ fitted to 


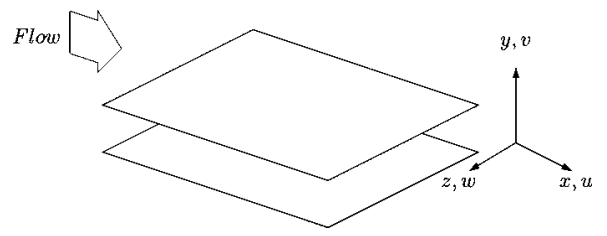

FIG. 2. The channel geometry. The coordinate axes are referred to as streamwise $x$, wall normal $y$, and spanwise $z$. The velocity components in $x$, $y$, and $z$ are denoted $u, v$, and $w$.

solutions of the Fokker-Planck equation for simple flows. The invariant-based optimal fitted (IBOF) closure, used for the turbulent flow calculations in Ref. 5, is similar to EBOF, except that it is formulated in terms of the tensor invariants. Both methods are proved to be of equal accuracy and are probably the best schemes available at present. ${ }^{14}$

We have constructed three EBOF schemes, fitting solutions of the Fokker-Planck equation at three different rotary Peclet numbers $\mathrm{Pe}_{r}=50,200$, and 800. The details and results of this procedure are given in the Appendix. We refer to these schemes as EBOF50, EBOF200, and EBOF800. In Fig. 1 we show the performance of these closures in predicting the shear viscosity [Eq. (14)] as a function of $\mathrm{Pe}_{r}$. The solid line shows the exact solution computed with the FokkerPlanck equation. In the weak flow regime $\mathrm{Pe}_{r}<10$, the closures produce the correct result $\eta_{x y}=1 / 3$. For larger $\mathrm{Pe}_{r}$ the closures produce a power law $\eta_{x y} \sim \mathrm{Pe}_{r}^{n}$ with a smaller exponent $n \approx-0.45$ than the exact value of $-1 / 3$. Eventually at even larger $\mathrm{Pe}_{r}$ the closures produce qualitatively wrong results; $\eta_{x y}$ deviates from the power law and approaches a constant.

It is likely that the EBOF closure performs best for flow problems in which $\mathrm{Pe}_{r}$ matches $\mathrm{Pe}_{r}$ used in the fit solutions. With increasing $\mathrm{Pe}_{r}$ these solutions become increasingly expensive with the spherical harmonics method. Therefore, for relatively large $\mathrm{Pe}_{r}$ a particle method like that in Ref. 6 would be more suitable.

The subject of this paper is the performance of the EBOF closure in two-way coupled turbulent fiber suspension channel flow. With increasing $\mathrm{Pe}_{r}$ the convergence of the expansion [Eq. (9)] decreases, effectively increasing the importance of the $l=4$ modes and the closure. Therefore the simulations are carried out at $\mathrm{Pe}_{r}$ as large as numerically feasible. A difficulty in turbulent flow is the wide variety of Peclet numbers, making the choice of $\mathrm{Pe}_{r}$ used in the fit data an important issue. The effect of this choice is studied in Sec. VI A.

\section{NUMERICAL METHOD FOR THE TURBULENT CHANNEL FLOW}

\section{A. Eulerian method}

We numerically solve the incompressible Navier-Stokes equations complemented by the divergence of the fiber stress [Eq. (5)] in the channel geometry (Fig. 2), $\frac{\partial \boldsymbol{u}}{\partial t}+\boldsymbol{u} \cdot \nabla \boldsymbol{u}=-\frac{1}{\rho} \nabla p+\nu \nabla^{2} \boldsymbol{u}+\frac{1}{\rho} \nabla \cdot \boldsymbol{\tau}, \quad \boldsymbol{\nabla} \cdot \boldsymbol{u}=0$.

Here $p$ is the pressure, $\rho$ is the mass density, and $\nu=\mu / \rho$ is the kinematic viscosity. The fiber stress is computed using two methods: the Fokker-Plank equation (4) and the moment equation (16) closed with EBOF200 [Eq. (A8)]. The flow is driven between two parallel no-slip walls by means of a constant pressure gradient $-\partial \bar{p} / \partial x$. Periodic boundary conditions are imposed in the homogeneous directions $x$ and $z$. Wall normal derivatives of $f$ and its moments are zero at the walls.

We use a pseudospectral flow solver, which is similar to the one used in Ref. 1. Spatial derivatives are computed with a Fourier basis for the homogeneous directions and a secondorder central finite differences scheme for the wall normal direction. Time integration is achieved with the second-order explicit Adams-Bashforth scheme. Conservation of mass is ensured using a standard projection method. Poisson's equation is transformed to Fourier space in the homogeneous directions and a tridiagonal solver is used for the resulting three band matrices. The variables are discretized on a nonequidistant staggered mesh. Fiber orientation, pressure, and the velocity components in the homogeneous directions are defined in the cell centers. The wall normal velocity component is defined on the cell faces.

The concentration parameter $\alpha=10$ [Eq. (7)]. Assuming fibers with $r \sim 100$, this implies $n L^{3} \sim 50$, with $L$ the fiber length and $n$ the number of fibers per unit volume. This parameter value corresponds to a semidilute suspension, meaning that fiber-fiber interactions are important. ${ }^{9}$ For simplicity we do not take these effects into account. Our main focus is the fourth-order moment closure. The Reynolds number

$$
\operatorname{Re}=\frac{U_{\tau} H}{\nu}=449,
$$

where $U_{\tau}$ is the friction velocity, $U_{\tau}=\sqrt{(-\partial \bar{p} / \partial x)(H / \rho)}$, and $H$ is the full channel height. The effective Reynolds number

$$
\operatorname{Re}_{\text {eff }}=\frac{U_{\tau} H}{\nu_{\text {eff }}}
$$

is based on the effective viscosity at the wall $\nu_{\text {eff }}$,

$$
\nu_{\mathrm{eff}}=\frac{\nu \frac{\partial \bar{u}}{\partial y}+\frac{1}{\rho} \overline{\tau_{x y}}}{\frac{\partial \bar{u}}{\partial y}}(y=0),
$$

where $\tau_{x y}$ is the fiber shear stress and the bar signifies averaged. The effective Reynolds number is around 250 being somewhat different for the Fokker-Planck simulation and the moment simulation. We use this relatively low value for $\mathrm{Re}_{\mathrm{eff}}$ due to two computational restrictions. First, the number of spatial grid points, necessary to resolve all the scales in the turbulent flow, increases as $\mathrm{Re}_{\mathrm{eff}}^{9 / 4}$. To solve the Fokker-Planck equation an orientation resolution is required on top of the spatial resolution. Second, the time step needed to ensure a stable simulation of the Fokker-Planck equation is inversely proportional to the maximum shear rate in the channel, 
which in turn is proportional to the Reynolds number.

As argued in Sec. IV B we aim at testing the closure at $\mathrm{Pe}_{r}$ as large as possible. With increasing $\mathrm{Pe}_{r}$ the gradients in the orientational part of $f$ become steeper, and more spherical basis functions are required to accurately describe $f$. We decided that for $N=12$ (91 orthogonal modes) the computation is still tractable. Allowing a maximum truncation error of $1 \%$, it follows from Fig. 1 that we can use a maximum value of $\mathrm{Pe}_{r}=50$. Since the largest shear rates occur at the wall, this Peclet number is based on the mean shear rate at the wall,

$$
\mathrm{Pe}_{r}=\frac{U_{\tau}^{2}}{\nu_{\mathrm{eff}} d_{r}} .
$$

Note that this is a global definition. The local Peclet number [Eq. (13)] varies over space and time. The spatial Peclet number is defined as

$$
\mathrm{Pe}_{s}=\frac{U_{\tau} H}{d_{s}} .
$$

Since $\mathrm{Pe}_{s} / \mathrm{Pe}_{r} \sim(H / L)^{2} / \mathrm{Re}_{\text {eff }}$ (Ref. 9), realistic $\mathrm{Pe}_{s}$ values are relatively large. The characteristic scales of the spatial variation of $f$ decrease with $\mathrm{Pe}_{s}$, analogous to that of passive scalars. ${ }^{15}$ This causes $f$ to be very detailed spatially, requiring an unfeasible number of grid points. Since under resolving results in numerical instabilities, we have to use an artificial small value for $\mathrm{Pe}_{s}$. Tests have shown that our numerical solution is stable and well behaved up to $\mathrm{Pe}_{s}$ $=125$, which is the value used. This value is comparable to that used in other numerical studies on polymer moment equations (see, for instance, Ref. 1). The effect of a relatively large artificial diffusivity on the solution of the FokkerPlanck equation will be investigated in depth in a subsequent work. Here we note that these effects are most likely to be similar to those observed in polymer moment equations, where it is generally found that the numerical solution does not change significantly for values larger than $\mathrm{Pe}_{s}$ $\sim \operatorname{Re} \Delta t \nu_{\text {eff }} / \Delta x^{2}$. Here $\Delta x$ is the typical mesh dimension and $\Delta t$ the time step. So-called local artificial diffusion schemes have been developed to minimize the diffusion influence. ${ }^{5}$ This technique could not be used here since it yields local det $(\langle\boldsymbol{p} \boldsymbol{p}\rangle)<0$, causing instabilities in the Fokker-Planck solution.

The channel dimensions and number of grid points are $5 H \times H \times 2.5 H$ and $96 \times 96 \times 96$ in the streamwise $x$, wall normal $y$, and spanwise $z$ directions. The grid is nonuniform in $y$ with a $4 \%$ increase in cell-volume per cell away from the wall. The grid-cell size in $\nu_{\text {eff }} / U_{\tau}$ units (wall units) at the wall and in the channel center are $13.0 \times 1.2 \times 6.5$ and 13.0 $\times 4.7 \times 6.5$. Except in the wall normal direction close to the wall, the grid-cell size matches the one used in Ref. 16, which is generally accepted as resolving all relevant turbulent scales. A time step of $\Delta t=5 \times 10^{-5} H / U_{\tau}$ is used. After the simulation is statistically converged, 200 samples are collected during the time interval of $t=5 H / U_{\tau}$. A Newtonian reference case is computed using the same parameters except for $\alpha=0$ and $\mathrm{Re}=\mathrm{Re}_{\mathrm{eff}}=250$.

The Message Passing Interface-parallelized FORTRAN computer codes were run on AMD Opteron $2.0 \mathrm{GHz}$ proces- sors. For the Fokker-Planck simulation, seven processors were used. Together they took 300 seconds per time step. The moment simulation was run on a single processor, taking 30 seconds per time step.

\section{B. Lagrangian method}

In the Appendix three different EBOF schemes are derived at three different $\mathrm{Pe}_{r}$. We determined the relative performance of the different EBOF schemes. These preliminary tests were done at low costs by performing one-way coupled Lagrangian simulations, eliminating the computation of the spatial derivatives in the Fokker-Planck equation. The reduced cost allows a larger $\mathrm{Pe}_{r}$ so that the influence of this parameter on closure performance can be studied. Therefore, two rotary Peclet numbers are considered, $\mathrm{Pe}_{r}=50$ and $\mathrm{Pe}_{r}$ $=200$. The Reynolds number is $\operatorname{Re}=360$.

To solve the Fokker-Planck and moment equations along the trajectories of fluid particles, fluid velocity and velocity gradient values at the position of the fluid particles are interpolated. The interpolation scheme uses the Fourier basis in the homogeneous directions and a third-order polynomial fit in wall normal direction. The position of the fluid particles is advanced in time using the second-order Adams-Bashforth scheme. We use $N=32$ corresponding to 561 orthogonal basis functions. The domain size and number of grid points are $3 H \times H \times 1.5 H$ and $96 \times 96 \times 96$. The grid in the wall normal direction is stretched, as described in Sec. V A. The grid-cell size in wall units at the wall and in the channel center are $11.3 \times 1.8 \times 5.7$ and $11.3 \times 6.7 \times 5.7$. The integration is carried out over 1000 fluid trajectories over a time interval of $t=10 H / U_{\tau}$ using a time step of $\Delta t=1.5 \times 10^{-5} H / U_{\tau}$. Statistics are computed over 1000 samples per trajectory.

\section{RESULTS}

\section{A. Lagrangian one-way coupled simulations}

Stresses computed with the Lagrangian method are given in Fig. 3. Only the streamwise normal stress component is considered, since the closure error in this component is largest. The stress is computed using $\alpha=1$ [Eq. (5)] and scaled with wall shear stress $\left(\rho U_{\tau}^{2}\right)$. Note that the stress shown in this figure is not coupled back to the equations of fluid motion, i.e., these simulations are one-way coupled. We use $\cdots,(\cdots)^{\prime}$, and $(\cdots)_{\text {rms }}$ to denote mean part, fluctuating part, and standard deviation. Wall distance is presented in wall units, where 250 wall units correspond to the full channel height.

With increasing $\mathrm{Pe}_{r}$ the stress decreases, which is most pronounced in the viscous sublayer $\left(y^{+}<10\right)$. This effect, also observed in Ref. 6, is due to shear thinning discussed in Sec. III B. It is found that the performance of the different closures depend on $\mathrm{Pe}_{r}$ and wall distance. At $\mathrm{Pe}_{r}=50$, EBOF50 produces the best results for $y^{+}>10$ in both the mean and standard deviations. For $y^{+}<10$ the mean is best captured by EBOF200, while EBOF800 produces the best standard deviation. Since the overall error for EBOF200 is smallest, this scheme is used for the two-way coupled simulations. Increasing $\mathrm{Pe}_{r}$ from 50 to 200, the closure error in 

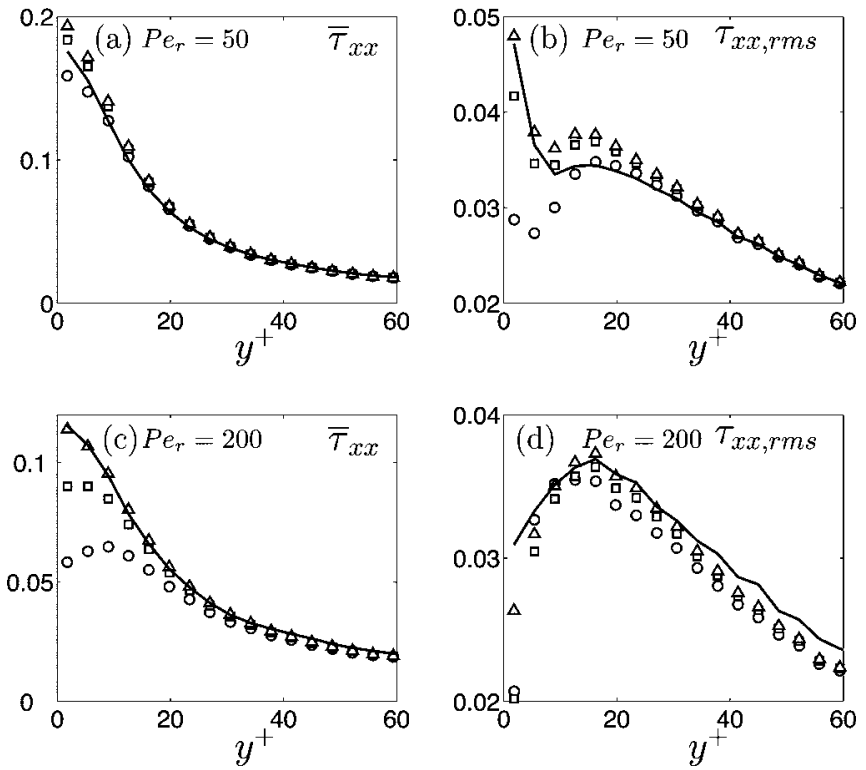

FIG. 3. Mean (left) and standard deviation (right) of streamwise normal stress component as a function of wall distance for Newtonian channel flow. Comparison between Fokker-Planck equation (-) and moment equation closed with EBOF50 (circles), EBOF200 (squares), and EBOF800 (triangles).

the stress standard deviation increases slightly. At $\mathrm{Pe}_{r}=200$, EBOF800 produces the best results for both the mean and standard deviations.

\section{B. Eulerian two-way coupled simulations}

Figures 4 and 5 show fiber orientation and fiber stress in
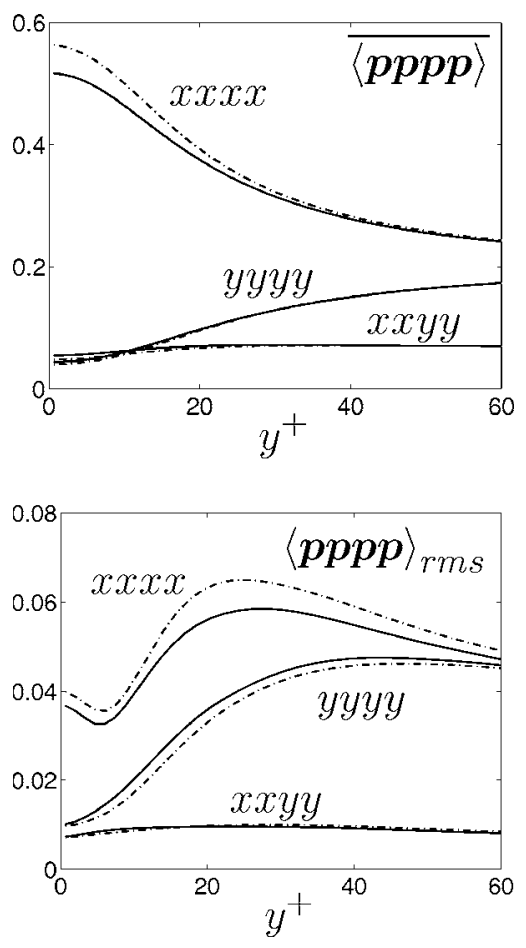

FIG. 4. Mean (top) and standard deviation (bottom) of fourth-order components of $f$. Comparison between Fokker-Planck equation (-) and moment equation closed with EBOF200 (-.).
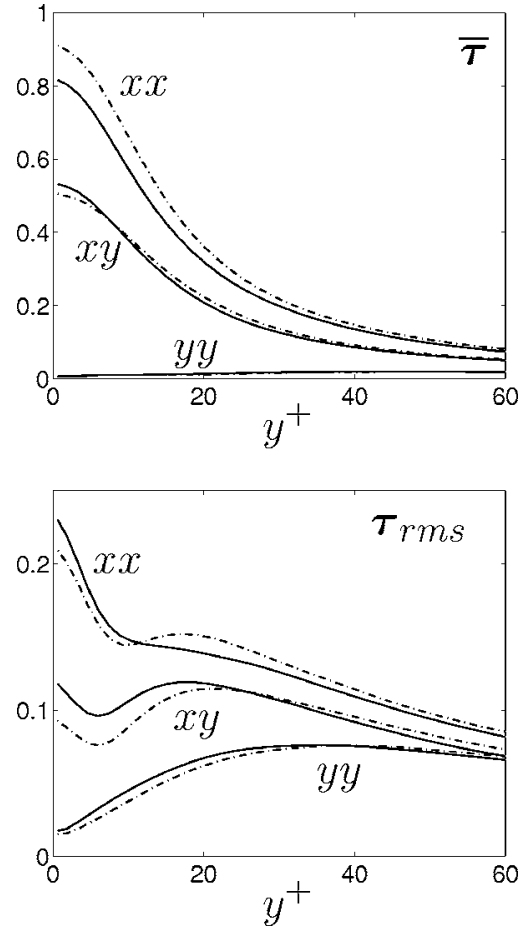

FIG. 5. Mean (top) and standard deviation (bottom) of stress components. Comparison between Fokker-Planck equation (-) and moment equation closed with EBOF200 (-.).

the two-way coupled turbulent channel flow simulation. In the viscous sublayer the flow is dominated by shear, and particles are strongly orientated in the streamwise direction. Here the moments of $f$ are highly anisotropic and deviate relatively little from their mean values. Further away from the wall turbulent fluctuations enhance the rms values and move the mean values towards isotropy. The moment equation closed with EBOF200 [Eq. (A8)] is compared to the direct computation of $f$. The agreement is good. The streamwise component of $\boldsymbol{p}$ is slightly overestimated and the fluctuations in $\boldsymbol{p}$ are somewhat underestimated. Errors in stress are $\sim 10 \%$ for both the mean and standard deviations.

Flow statistics scaled with $U_{\tau}, \rho$, and $\nu_{\text {eff }}$ are presented in Figs. 6-8, comparing Newtonian flow and non-Newtonian flow computed with the Fokker-Planck equation and the moment equation. The effective Reynolds numbers [Eq. (19)] are $\mathrm{Re}_{\text {eff }}=255$ for the Fokker-Planck simulation and $\mathrm{Re}_{\text {eff }}$ $=271$ for the moment simulation. This difference is because the closure predicts a lower fiber shear stress as compared to the exact Fokker-Planck result (see Fig. 1). It is noted that fiber statistics differ due to the slight difference in $\mathrm{Re}_{\text {eff }}$. Paschkewitz et al. investigated this issue and found the difference to be quite modest at low $\operatorname{Re}_{\text {eff }}$ values. ${ }^{5}$

Before discussing closure performance in more detail, we first compare the fiber suspension flow to the Newtonian flow. Figure 6 reveals that the fibers reduce the drag by 5\%, defined as the relative decrease in drag coefficient $C_{D}$,

$$
C_{D}=\left(\frac{U_{\tau}}{U_{b}}\right)^{2}
$$

where the bulk velocity $U_{b}$ equals the streamwise velocity averaged over the channel. Note that this reduced friction is 

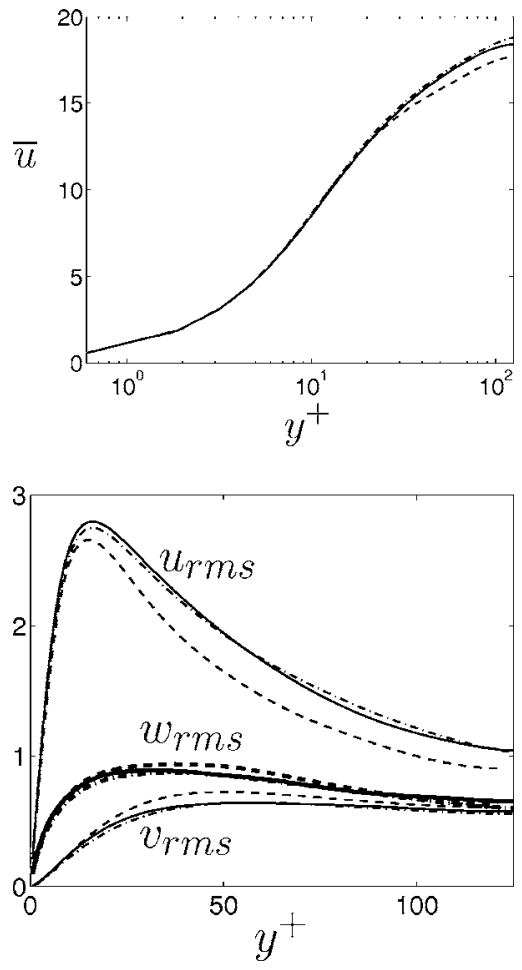

FIG. 6. Mean (top) and standard deviation (bottom) of the fluid velocity. Comparison between Fokker-Planck equation (-), moment equation closed with EBOF200 (--), and Newtonian (--).
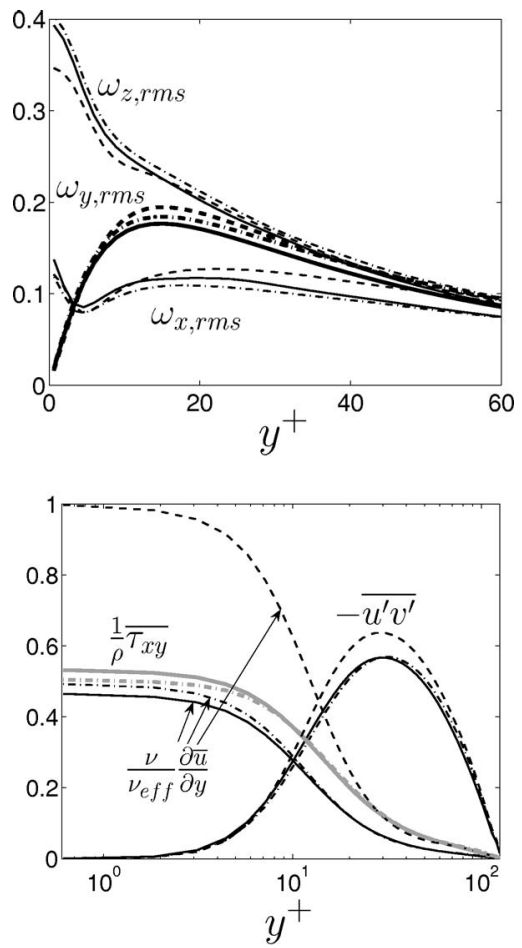

FIG. 7. (Top) standard deviation of vorticity. (Bottom) contributions to the averaged momentum equation [Eq. (24)]. Comparison between FokkerPlanck equation (-), moment equation closed with EBOF200 (-.), and Newtonian (--).
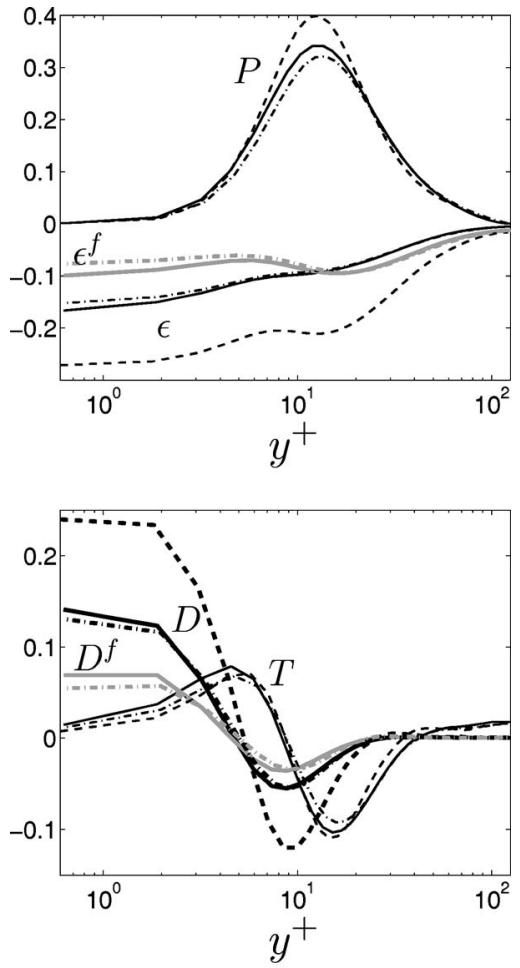

FIG. 8. Contributions to the averaged turbulent kinetic-energy equation [Eq. (25)]. Comparison between Fokker-Planck equation (-), moment equation closed with EBOF200 (-), and Newtonian (--).

measured at constant $\mathrm{Re}_{\text {eff. }}$. When compared at constant $\mathrm{Re}$ this suspension is drag increased. There are modest changes in the turbulent structures, typical for a "drag reduced" flow. The turbulent velocity intensity $\boldsymbol{u}_{\text {rms }}$ is increased in $x$ and reduced in $y$ and $z$. The turbulent vorticity intensity $\boldsymbol{\omega}_{r m s}$ is increased in $z$ and reduced in $x$ and $y$. The Reynolds shear stress $-\overline{u^{\prime} v^{\prime}}$ [Eq. (24)] and the production due to mean shear $P$ [Eq. (25)] are reduced.

The comparison between the Fokker-Planck equation and the moment equation reveals the following. The mean velocity and the turbulent velocity and vorticity intensities differ by less than $5 \%$. Also the terms in the averaged, nondimensionalized momentum equation,

$$
\frac{\nu}{\nu_{\mathrm{eff}}} \frac{\partial \bar{u}}{\partial y}-\overline{u^{\prime} v^{\prime}}+\overline{\tau_{x y}}=1-2 \frac{y}{\mathrm{Re}_{\mathrm{eff}}},
$$

and the terms in the averaged turbulent kinetic energy equation, ${ }^{17}$

$$
\begin{aligned}
& -2 \overline{u_{1}^{\prime} u_{2}^{\prime}} \frac{\partial \overline{u_{1}}}{\partial x_{2}}-\frac{\partial}{\partial x_{2}} \overline{u_{2}^{\prime}\left(u_{i}^{\prime} u_{i}^{\prime}+\frac{2 p^{\prime}}{\rho}\right)}+\frac{\nu}{\nu_{\mathrm{eff}} \frac{\partial^{2}}{\partial x_{2}^{2}} \overline{u_{i}^{\prime} u_{i}^{\prime}}} \\
& -2 \frac{\nu}{\nu_{\mathrm{eff}}} \overline{\frac{\partial u_{i}^{\prime}}{\partial x_{j}} \frac{\partial u_{i}^{\prime}}{\partial x_{j}}}+2 \frac{\partial}{\partial x_{2}} \overline{\tau_{i 2}^{\prime} u_{i}^{\prime}}-2 \tau_{i j}^{\prime} \frac{\partial u_{i}^{\prime}}{\partial x_{j}}=0, \\
& P+T+D+\epsilon+D^{f}+\epsilon^{f}=0,
\end{aligned}
$$

agree very well. A relatively large error is observed for $y^{+}$ $<5$, where the closure-predicted dissipation due to fiber stress $\epsilon^{f}$ and the diffusion due to fiber stress $D^{f}$ are $10 \%$ too small. These errors are related to the error in shear viscosity, 
as discussed in Sec. IV B. The lower $\epsilon^{f}$ near the wall is linked with the lower peak value of $P$ at $y^{+} \approx 15$.

\section{CONCLUSIONS}

Two-way coupled turbulent fiber suspension flow simulations using the Fokker-Planck equation and the EBOF closure are compared at low rotary Peclet number $\mathrm{Pe}_{r}=50$, being the ratio of wall shear and rotary diffusion. The EBOF predicted fiber stress, momentum budgets, and kineticenergy budgets are within $10 \%$ of the Fokker-Planck solution. A one-way coupled simulation at $\mathrm{Pe}_{r}=200$ shows a slight increase in closure error from which we anticipate that the error grows with $\mathrm{Pe}_{r}$. Since significant drag reduction occurs for $\mathrm{Pe}_{r}>10^{3}$, closure performance in drag reduced flow is still an open issue.

It is possible to construct an EBOF closure which is asymptotically correct for simple shear in the limit $\mathrm{Pe}_{r} \rightarrow \infty$ by imposing extra constraints on the parametric form, Eq. (A1). This would require an extension of the number of fit parameters. Although we have not done this here, we think that this will improve results in turbulent shear flows at large $\mathrm{Pe}_{r}$.

\section{ACKNOWLEDGMENTS}

This research has been partially supported through the PETROMAKS programme funded by The Research Council of Norway. The authors thankfully acknowledge the encouragement and inspiration provided by the late Professor F. T. M. Nieuwstadt who was instrumental in the early stages of this work. Discussions with Professor Michael Manhart are gratefully acknowledged.

\section{APPENDIX: THE EBOF CLOSURE}

The EBOF closure, due to Cintra and Tucker, ${ }^{7}$ relates the fourth moment to the second moment of the fiber orientation distribution function. The closure is a quadratic fit to "exact" solutions of the Fokker-Planck equation for several homogeneous cases. The closure is formulated in the principal frame of the second moment, which is also the principal frame of the fourth moment. In this frame $\langle\boldsymbol{p} \boldsymbol{p}\rangle$ has three nonzero values, two of which are independent owing to normalization [Eq. (2)]. Similarly $\langle\boldsymbol{p p p p}\rangle$ has nine nonzero values, six of which are unique due to symmetry and three are independent owing to normalization. The principle values of $\langle\boldsymbol{p} \boldsymbol{p}\rangle$ are denoted $a_{11}, a_{22}$, and $a_{33}$. The coordinates are such that $a_{11}$ $\geqslant a_{22} \geqslant a_{33}$. The principal values of $\langle\boldsymbol{p} \boldsymbol{p} \boldsymbol{p} \boldsymbol{p}\rangle$ are denoted $a_{i j k l}$. For simplicity the indices $i j k l$ of the six unique nonzero fourth-order components are abbreviated by $m^{\prime}$ as follows:

$$
\begin{array}{ccccccc}
m^{\prime}: & 1 & 2 & 3 & 4 & 5 & 6 \\
i j k l: & 1111 & 2222 & 3333 & 1122 & 1133 & 2233
\end{array}
$$

The closure expresses these six components as quadratic functions of $a_{11}$ and $a_{22}$,

$$
\begin{aligned}
a_{m^{\prime}}^{\text {closure }}= & C_{m^{\prime}}^{1}+C_{m^{\prime}}^{2} a_{11}+C_{m^{\prime}}^{3} a_{22}+C_{m^{\prime}}^{4} a_{11} a_{22} \\
& +C_{m^{\prime}}^{5} a_{11}^{2}+C_{m^{\prime}}^{6} a_{22}^{2} .
\end{aligned}
$$

The polynomial coefficients are obtained from a least-square fit minimizing

$$
\chi^{2}=\sum_{i=1}^{N_{\mathrm{DATA}}} \sum_{m^{\prime}=1}^{6}\left(a_{m^{\prime}}^{i}-a_{m^{\prime}}^{i, \text { closure }}\right)^{2}
$$

Here $N_{\text {DATA }}$ is the number of data points to which the closure is fitted, $a_{m^{\prime}}^{i}$ is the $i$ th data point obtained from the FokkerPlanck equation, and $a_{m^{\prime}}^{i, \text { closure }}$ is the corresponding value predicted by the closure [Eq. (A1)]. In addition to Eq. (A2) the normalization constraint is satisfied,

$$
\begin{aligned}
& a_{1^{\prime}}+a_{4^{\prime}}+a_{5^{\prime}}=a_{11}, \quad a_{2^{\prime}}+a_{4^{\prime}}+a_{6^{\prime}}=a_{22}, \\
& a_{3^{\prime}}+a_{5^{\prime}}+a_{6^{\prime}}=a_{33} .
\end{aligned}
$$

There are 36 fit coefficients $C_{m^{\prime}}^{k}, 18$ of which are independent owing to normalization. These are the coefficients for $m^{\prime}=1,2,3$. Differentiating Eq. (A2) to the independent coefficients and equating the results to zero yields a system of linear equations from which the independent coefficients are obtained. This procedure is applied to homogeneous solutions of the Fokker-Planck equation for several flow cases,

$\boldsymbol{\kappa}=\gamma\left(\begin{array}{lll}0 & 1 & 0 \\ 0 & 0 & 0 \\ 0 & 0 & 0\end{array}\right), \quad \boldsymbol{\kappa}=\frac{\gamma}{4 \sqrt{7}}\left(\begin{array}{ccc}-1 & 10 & 0 \\ 0 & -1 & 0 \\ 0 & 0 & 2\end{array}\right)$,

$\boldsymbol{\kappa}=\frac{\gamma}{\sqrt{13}}\left(\begin{array}{ccc}-1 & 1 & 0 \\ 0 & -1 & 0 \\ 0 & 0 & 2\end{array}\right), \quad \boldsymbol{\kappa}=\frac{\gamma}{2 \sqrt{3}}\left(\begin{array}{ccc}-1 & 0 & 0 \\ 0 & -1 & 0 \\ 0 & 0 & 2\end{array}\right)$

$\boldsymbol{\kappa}=\frac{\gamma}{2 \sqrt{3}}\left(\begin{array}{ccc}1 & 0 & 0 \\ 0 & 1 & 0 \\ 0 & 0 & -2\end{array}\right), \quad \boldsymbol{\kappa}=\frac{\gamma}{2 \sqrt{26}}\left(\begin{array}{ccc}-1 & 0 & 10 \\ 0 & 1 & 0 \\ 0 & 0 & 0\end{array}\right)$

$\boldsymbol{\kappa}=\frac{\gamma}{\sqrt{5}}\left(\begin{array}{ccc}-1 & 0 & 1 \\ 0 & 1 & 0 \\ 0 & 0 & 0\end{array}\right), \quad \boldsymbol{\kappa}=\frac{\gamma}{4}\left(\begin{array}{ccc}1 & 0 & 2 \\ 0 & 1 & 0 \\ 0 & 0 & -2\end{array}\right)$

$\boldsymbol{\kappa}=\frac{\gamma}{\sqrt{37}}\left(\begin{array}{ccc}1 & 0 & 5 \\ 0 & 1 & 0 \\ 0 & 0 & -2\end{array}\right)$

For each case the evolution of the distribution is calculated using $N=64$ ( 2145 basis functions), over a time interval of $\gamma t=20$, starting from an isotropic fiber orientation. The system of ordinary differential equations [Eq. (11)] can be written as 


$$
\frac{\partial \boldsymbol{A}}{\partial t}=\boldsymbol{M} \cdot \boldsymbol{A},
$$

with $\boldsymbol{A}$ the vector of expansion coefficients and $\boldsymbol{M}$ the matrix representation of the advection and diffusion operator. For steady flow conditions the solution reads

$$
\boldsymbol{A}(t)=\boldsymbol{T} \cdot \exp (\boldsymbol{\Lambda} t) \cdot \boldsymbol{T}^{-1} \cdot \boldsymbol{A}(t=0),
$$

with $\boldsymbol{T}$ the matrix containing the eigenvectors of $\boldsymbol{M}$ and $\boldsymbol{\Lambda}$ the diagonal matrix containing the eigenvalues of $\boldsymbol{M}$. The closure is fitted to 100 samples per case, taken equidistant in time. This yields a total of $N_{\text {DATA }}=900$. We have derived three closures at three different rotary Peclet numbers numbers: $\mathrm{Pe}_{r}=50, \mathrm{Pe}_{r}=200$, and $\mathrm{Pe}_{r}=800$, referred to as EBOF50, EBOF200, and EBOF800. The resulting fit coefficients $C_{m^{\prime}}^{k}$ are given below:

\begin{tabular}{rrrrrrrr}
\multicolumn{7}{c}{ EBOF50: } & \\
& $k$ & 1 & 2 & 3 & 4 & 5 & 6 \\
$m^{\prime}$ & & & & & & \\
1 & -0.036978 & 0.696435 & -0.219487 & 0.050781 & 0.314456 & 0.317181 \\
2 & 0.000996 & -0.038726 & 0.332232 & 0.153772 & 0.025545 & 0.720341 \\
3 & 1.191223 & -1.968742 & -2.146077 & 1.690789 & 0.770213 & 0.974192
\end{tabular}

\section{EBOF200:}

$\begin{array}{rrrrrrrr} & k & 1 & 2 & 3 & 4 & 5 & 6 \\ m^{\prime} & & & & & & \\ 1 & 0.023383 & 0.483130 & -0.313080 & 0.249338 & 0.486620 & 0.333032 \\ 2 & 0.065082 & -0.241884 & 0.213848 & 0.368282 & 0.173314 & 0.756985 \\ 3 & 1.238745 & -2.106540 & -2.264359 & 1.869141 & 0.866054 & 1.044024,\end{array}$

\begin{tabular}{rrrrrrrr}
\multicolumn{7}{c}{ EBOF800: } \\
& $k$ & 1 & 2 & 3 & 4 & 5 & 6 \\
$m^{\prime}$ & & & & & & \\
1 & 0.065916 & 0.354266 & -0.392032 & 0.372329 & 0.582099 & 0.362986 \\
2 & 0.102926 & -0.346018 & 0.132738 & 0.485745 & 0.244133 & 0.789574 \\
3 & 1.244530 & -2.132195 & -2.275148 & 1.899767 & 0.888406 & 1.047289.
\end{tabular}

Per closure the FORTRAN computer code took 75 min on one AMD Opteron $2.0 \mathrm{GHz}$ processor.

${ }^{1}$ P. K. Ptasinski, B. J. Boersma, F. T. M. Nieuwstadt, M. A. Hulsen, B. H. A. van den Brule, and J. C. R. Hunt, "Turbulent channel flow near maximum drag reduction: simulations, experiments and mechanisms," J. Fluid Mech. 490, 251 (2003).

${ }^{2}$ J. S. Paschkewitz, C. D. Dimitropoulus, Y. X. Hou, V. S. R. Somandepalli, M. G. Mungal, E. S. G. Shaqfeh, and P. Moin, "An experimental and numerical investigation of drag reduction in a turbulent boundary layer using a rigid rodlike polymer," Phys. Fluids 17, 085101 (2005).

${ }^{3}$ W. D. McComb and K. T. J. Chan, "Laser-Doppler anemometer measurements of turbulent structure in drag-reducing fibre suspensions," J. Fluid Mech. 152, 455 (1985).

${ }^{4}$ A. Gyr and H. W. Bewersdorff, Drag Reduction of Turbulent Flows by Additives (Kluwer, London (1995).

${ }^{5}$ J. S. Paschkewitz, Y. Dubief, C. D. Dimitropoulus, E. S. G. Shaqfeh, and P. Moin, "Numerical simulation of turbulent drag reduction using rigid fibres," J. Fluid Mech. 518, 281 (2004).

${ }^{6} \mathrm{M}$. Manhart, "Rheology of suspension of rigid-rod like particles in turbulent channel flow," J. Non-Newtonian Fluid Mech. 112, 269 (2003).

${ }^{7}$ J. S. Cintra and C. L. Tucker, "Orthotropic closure approximations for flow-induced fiber orientation,” J. Rheol. 391095 (1995).

${ }^{8}$ J. S. Paschkewitz, Y. Dubief, and E. S. G. Shaqfeh, "The dynamic mechanism for turbulent drag reduction using rigid fibers based on Lagrangian conditional statistics," Phys. Fluids 17, 063102 (2005).
${ }^{9}$ M. Doi and S. F. Edwards, The Theory of Polymer Dynamics (Clarendon, Oxford, 1986).

${ }^{10}$ W. E. Stewart and J. P. Sorensen, "Hydrodynamic interaction effects in rigid dumbbell suspensions. II. Computations for steady shear flow," Trans. Soc. Rheol. 16, 1 (1972).

${ }^{11}$ M. Abramowitz and A. Stegun, Handbook of Mathematical Functions (Dover, New York, 1965).

${ }^{12} \mathrm{P}$. N. Schwarztrauber, "On computing the points and weights for GaussLegendre quadrature," Solid State Commun. 24, 945 (2002).

${ }^{13}$ C. V. Chaubal and L. G. Leal, "A closure relation for liquid-crystalline polymer models based on parametric density estimation," J. Rheol. 42, 17 (1997).

${ }^{14}$ D. Chung and T. Kwon, "Invariant-based optimal fitting closure approximation for flow-induced fiber orientation," J. Rheol. 22, 636 (2002).

${ }^{15}$ G. K. Batchelor, "Small-scale variation of convected quantities like temperature in turbulent fluid. Part 1. General discussion and the case of small conductivity," J. Fluid Mech. 5, 113 (1959).

${ }^{16}$ J. Kim, P. Moin, and R. D. Moser, "Turbulence statistics in fully developed channel flow at low Reynolds number," J. Fluid Mech. 177, 133 (1987).

${ }^{17}$ H. Tennekes and J. L. Lumley, A First Course in Turbulence (MIT Press, Cambride, MA, 1973). 